\title{
Model elastomeric networks prepared by selectively cross- linking polydimethylsiloxane chains having known amounts of reactive side chains
}

\author{
J. R. Falender ${ }^{\text {a) }}$ and G. S. Y. Yeh \\ Department of Chemical Engineering and Macromolecular Research Center, University of Michigan, Ann
} Arbor, Michigan 48109

J. E. Mark

Department of Chemistry and Polymer Research Center, University of Cincinnati, Cincinnati, Ohio 45221 (Received 28 January 1979)

One of the most important issues in the area of rubberlike elasticity concerns the relationship between the modulus of an elastomeric material and its degree of cross-linking or the average molecular weight $M_{c}$ be tween cross-links in the network structure. Molecular theories of rubberlike elasticity ${ }^{1,2}$ provide predictions of this relationship, of course, for any value of the network functionality $\phi$. Relevant experimental data are rather scarce, however, because of the difficulty in obtaining a reliable independent value of $M_{c}$ for a network.

Some progress is now being made on the experimental side of this problem, through the preparation and study of "model" polymer networks, i.e., those of controlled and well characterized structure. In some earlier studies of this type, model networks were prepared by endlinking hydroxyl-terminated polydimethylsiloxane (PDMS) chains using either a tetrafunctional ${ }^{3}$ or trifunctional $^{4}$ silicate. Values of the modulus or reduced stress $\left[f^{*}\right]$ of the networks were extrapolated to reciprocal elongation $\alpha^{-1}$ equal to zero to obtain the constant $2 C_{1}$ given by theory as

$$
2 C_{1}=A_{\phi} \rho k T M_{c}^{-1},
$$

where $\rho$ is the density of the network. The structure factor $A_{\phi}$ is equal to $1-2 / \phi, 1,2$ and should thus range from 0.33 for the minimum functionality of three, to 1.00 for an indefinitely large functionality. Theory and experiment were found to be in very good agreement for both tetrafunctional and trifunctional PDMS networks ${ }^{3,4}$; specifically, in the tetrafunctional case, the experimental and theoretical values of $A_{4}$ were 0.65 and 0.50 , respectively.

Another chemical reaction suitable for preparing PDMS model networks involves the highly selective reaction of a vinyl group on the polymer with an active hydrogen atom on a silane cross-linking agent. This reaction goes essentially to completion with no side reactions, isomerization, or byproducts. ${ }^{5}$ It was used in the present study to prepare another type of model PDMS network with which to test further the theoretical predictions described above.

The polymer employed consisted of dimethylsiloxane chains having a molecular weight of $3.0 \times 10^{5}$; they contained $0.57 \mathrm{~mol} \%$ randomly incorporated methyl vinyl units (a concentration which corresponds to an average spacing of $M_{c}=13.0 \times 10^{3} \mathrm{~g} \mathrm{~mol}^{-1}$ between cross-linking sites). The silane cross-linking agent, $\mathrm{Si}\left[\mathrm{OSi}\left(\mathrm{CH}_{3}\right)_{2} \mathrm{H}\right]_{4}$, was present to an extent corresponding to a $2: 1$ molar ratio between active hydrogen atoms and vinyl groups, thus giving cross-links of average functionality four. The reaction was carried out for a period of one day at $95{ }^{\circ} \mathrm{C}$ with chloroplatinic acid as catalyst, and the resulting network was extracted, dried, and studied in elongation at $25^{\circ} \mathrm{C} . .^{3,4}$ The stress-strain isotherm, shown in Fig. 1, gave $2 C_{1}=0.111 \mathrm{~N} \mathrm{~mm}^{-2}$ upon least-squares analysis. This corresponds to $A_{4}=0.59$, which is in excellent agreement with the previous experimental result $(0.65)^{3}$ and with the appropriately weighted theoretical prediction $(0.53)$ for an average functionality of four. ${ }^{1}$

It is important to point out that the above agreement between theory and experiment is obtained without postulating contributions to the modulus from interchain entanglements, i.e., entanglements which involve chain segments relatively remote from their junction points. (This is in direct contradiction to Macosko's conclusion ${ }^{5}$ that trapped entanglements must be taken into account in order to explain the magnitude of the modulus of a PDMS network; the reason for this disagreement is not known at the present time.) The present results thus support the idea that such entanglements may be much less important with regard to the equilibrium elastic modulus than they are in the case of dynamic, viscoelastic properties. Similar results have also been obtained on other polymers, including those judged to be more "highly entangled" than PDMS on the basis of their values of the plateau modulus in the un-cross-linked state. ${ }^{6}$ For ex-

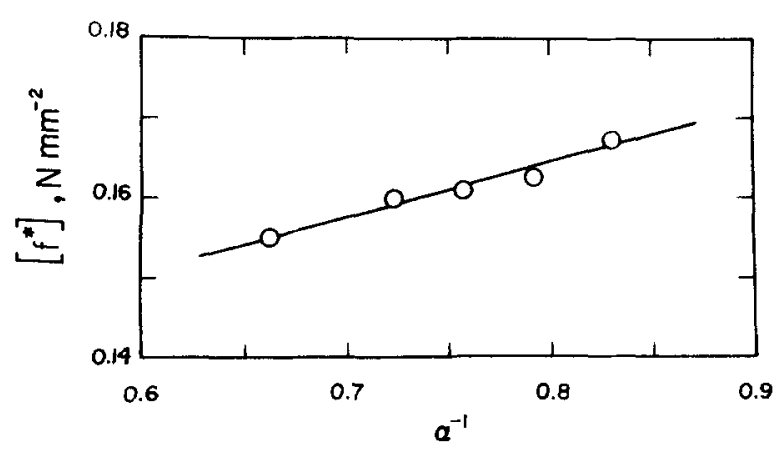

FIG. 1. The stress-strain isotherm of the model PDMS network at $25^{\circ} \mathrm{C}$. 
ample, Kraus and Moczvgemba ${ }^{7}$ studied end-linked model networks of polybutadiene (PBD) in which $\phi$ may have been as low as four but was probably six or higher. In the nine experiments pertinent here, they find the structure factor to be $0.64 \pm 0.15$, with a maximum value of 0.82 . These results are also in good agreement with the theoretical range $0.50-0.75$ for $\phi$ in the range four to eight. In a related study, ${ }^{8}$ Ono and co-workers have shown that the modulus (and tensile strength) of the PBD networks they investigated approached zero as $M_{c}$ increased, which also suggests the absence of large contributions from interchain entanglements. The same behavior has been reported for model networks prepared from PDMS and from polystyrene. ${ }^{9}$ In addition, Allen and co-workers ${ }^{10}$ report $A_{4} \simeq 0.7$ for model networks prepared from polystyrene and from polypropylene oxide. Some relevant measurements ${ }^{11}$ have also been reported on thermosetting unsaturated polyester networks. In the range of low degree of cross-linking (where the networks are still elastomeric), $A_{4}$ was again found to be approximately 0.5. Finally, some recent measurements ${ }^{12}$ on methacrylamide networks suggest a value of the structure factor of approximately 0.6.

Graessley and co-workers ${ }^{13}$ have, however, obtained rather different results in some electron-beam irradiation studies in which the gel fraction of PBD was determined as a function of radiation dose. They interpret these gelation results to give values of the structure factor ranging from 2 to 7 , and attribute these unusually large values to interchain entanglements. It should be noted, however, that the attempted interpretation of such results requires that the cross-links be introduced in a highly random manner. Such randomness may not occur in the case of cross-linking with high energy electrons or $\gamma$ radiation, because of collision cascading ${ }^{14}$ resulting in highly localized absorption of the quantum of radiation energy. ${ }^{15-17}$ Nonrandomness would necessitate a higher dosage in order to generate a coherent, macroscopic network because of the isolation of more highly crosslinked "spurs" or "tracks" by intervening material that had been little affected by the radiation. ${ }^{17}$ The experimentally observed gel point would therefore be delayed. Increasing the dosage so as to obtain a network sufficiently cross-linked for elasticity measurements could largely overcome the microheterogeneities prevalent at the gel point, and the elastic properties of the network would closely reflect the total number of cross-linkages present. Delay in the gel point due to microheterogeneities in the cross-linking would therefore lead to a fictitiously low estimate of the cross-linking efficiency, and hence of the degree of cross-linking in the samples ${ }^{13}$ prepared for the elasticity measurements. ${ }^{17}$ This nonrandomness could thus be the origin of the unusually large values obtained for the structure factor for networks prepared by this technique.

It is a pleasure to acknowledge the financial support provided by the Polymer Program in the Division of Materials Research of the National Science Foundation, and the sabbatical leave granted J.R.F. by the Dow Corning Corporation.

a) Present address: Dow Corning Corporation, Midland, Michigan 48640 .

${ }^{1}$ P. J. Flory, Proc. R. Soc. London Ser. A 351, 351 (1976);

J. Chem. Phys. 66, 5720 (1977).

${ }^{2}$ W. W. Graessley, Macromolecules 8, 186 (1975).

${ }^{3}$ J. E. Mark and J. L. Sullivan, J. Chem. Phys. 66, 1006 (1977).

4J. E. Mark, R. R. Rahalkar, and J. L. Sullivan, J. Chem. Phys. 70, 1794 (1979).

${ }^{5}$ E. M. Valles and C. W. Macosko in Chemistry and Properties of Crosslinked Polymers, edited by S. S. Labana (Academic, New York, 1977); Macromolecules (to be published).

${ }^{6} \mathrm{~J}$. D. Ferry, Viscoelastic Properties of Polymers, 2nd ed. (Wiley, New York, 1970).

${ }^{7}$ G. Kraus and G. A. Moczvgemba, J. Polymer Sci. Part A 2, 277 (1964).

${ }^{8} \mathrm{~K}$. Ono, H. Shimada, T. Nishimura, S. Yamashita, H. Okamoto, and Y. Minoura, J. Appl. Polym. Sci. 21, 3223 (1977).

${ }^{9}$ P. Rempp, J. Herz, G. Hild, and C. Picot, Pure Appl. Chem. 43, 77 (1975).

${ }^{10}$ D. J. Walsh, G. Allen, and G. Ballard, Polymer 15, 366 (1974);-G. Allen, P. A. Holmes, and D. J. Walsh, Faraday Discuss. Chem. Soc. 57, 19 (1974); G. Allen, P. L. Egerton, and D. J. Walsh, Polymer 17, 65 (1976).

${ }^{11}$ W. D. Cook, Eur. Polym. J. 14, 721 (1978).

${ }^{12}$ K. Ulbrich, K. Dušek, M. Ilavsky, and J. Kopeček, Eur. Polym. J. 14, 45 (1978).

${ }^{13}$ L. M. Dossin and W. W. Graessley, Macromolecules 12, 123 (1979); L. M. Dossin, D. S. Pearson, and W. W. Graessley, Abstracts of Intemational Rubber Conference, Kiev, 1978.

14“"Radiation Effects on Solid Surfaces," Adv. Chem. Ser. 158 (1976).

${ }^{15}$ I. W. Shepherd, Rep. Prog. Phys. 38, 565 (1975).

${ }^{16}$ T. -K. Su and J. E. Mark, Macromolecules 10, 120 (1977).

${ }^{17} \mathrm{P}$. J. Flory (private communication). 